Ophthalmologe 2022 $\cdot 119: 577-581$ https://doi.org/10.1007/s00347-021-01551-w Eingegangen: 1. Juli 2021 Überarbeitet:22. September 2021 Angenommen: 15. November 2021 Online publiziert: 18. Dezember 2021 ๑) Der/die Autor(en) 2021

\section{Zusammenarbeit mit klinischen Auftragsforschungsinstituten}

\section{Vorstellung eines Fragebogens für klinische Studienzentren}

\title{
Zusammenfassung
}

Klinische Studien mit Arzneimitteln oder Medizinprodukten stellen zunehmend komplexe Anforderungen an Sponsoren und beteiligte Zentren. In den letzten 2 Jahrzehnten delegieren Sponsoren regulatorische sowie organisatorische Studienaufgaben zunehmend an medizinische Auftragsinstitute (engl. Clinical Research Organisation [CRO]). In der Regel sind diese Unternehmen die Hauptschnittstelle für die Zusammenarbeit mit den beteiligten Studienzentren. Hauptzweck der Mitwirkung ist die Unterstützung der Studienzentren zur Erzielung einer maximalen Studienqualität. Die in der Arbeitsgemeinschaft DOG Klinische Studienzentren verbundenen Studienzentren beobachten unterschiedliche Erfahrungen in der Zusammenarbeit mit CROs. Solche Erfahrungen sollen künftig systematisch an den beteiligten Zentren erfasst und vom Leiter der klinischen Studie ausgewertet werden. Die Spiegelung dieser Erfahrungen an die jeweiligen Auftragsinstitute und die sie beauftragenden Sponsoren kann in der Zukunft zur Qualität der Unterstützung durch CROs und damit zur Studienqualität beitragen. Die vorliegende Arbeit stellt vor, welche Bereiche der Zusammenarbeit im Fragebogen erfasst und analysiert werden.

\section{Schlüsselwörter}

Klinische Studien · CRO · DOG · Studienqualität · Fragebogen

\section{Zusatzmaterial online}

Zusätzliche Informationen sind in der Online-Version dieses Artikels (https:// doi.org/10.1007/s00347-021-01551-w) enthalten.

\section{Wachsende Bedeutung medizinischer Auftragsinstitute (CROs)}

In den letzten 20 Jahren hat sich das Gefüge der an einer klinischen Studie mit Arzneimitteln oder Medizinprodukten beteiligten Partner stark verändert. Wurden zuvor die Aufgaben der Studieneinreichung und Vorbereitungen des Studienstarts sowie die Zusammenarbeit mit den beteiligten
Studienzentren in der laufenden Studie einschließlich des Monitorings noch von spezialisierten Abteilungen und Mitarbeitern seitens der Sponsoren selbst wahrgenommen, so änderte sich dies im genannten Zeitraum kontinuierlich, und es fand eine zunehmende Delegation dieser Aufgaben und Arbeitsbereiche an externe Anbieter statt. Heutzutage ist der Bereich der regulatorischen und organisatorischen Aufgaben vor, während und nach einer klinischen Studie weitestgehend von industriellen Sponsoren an medizinische Auftragsinstitute delegiert.

Seit der 12. Arzneimittelgesetz(AMG)Novelle ist die Unterstützung durch CROs sogar verpflichtend, wenn der Sponsor der Studie seinen Sitz außerhalb der Europäi- 
Welche Dienstleistungen vergeben Sie in der Regel extern? (Outsourcing)

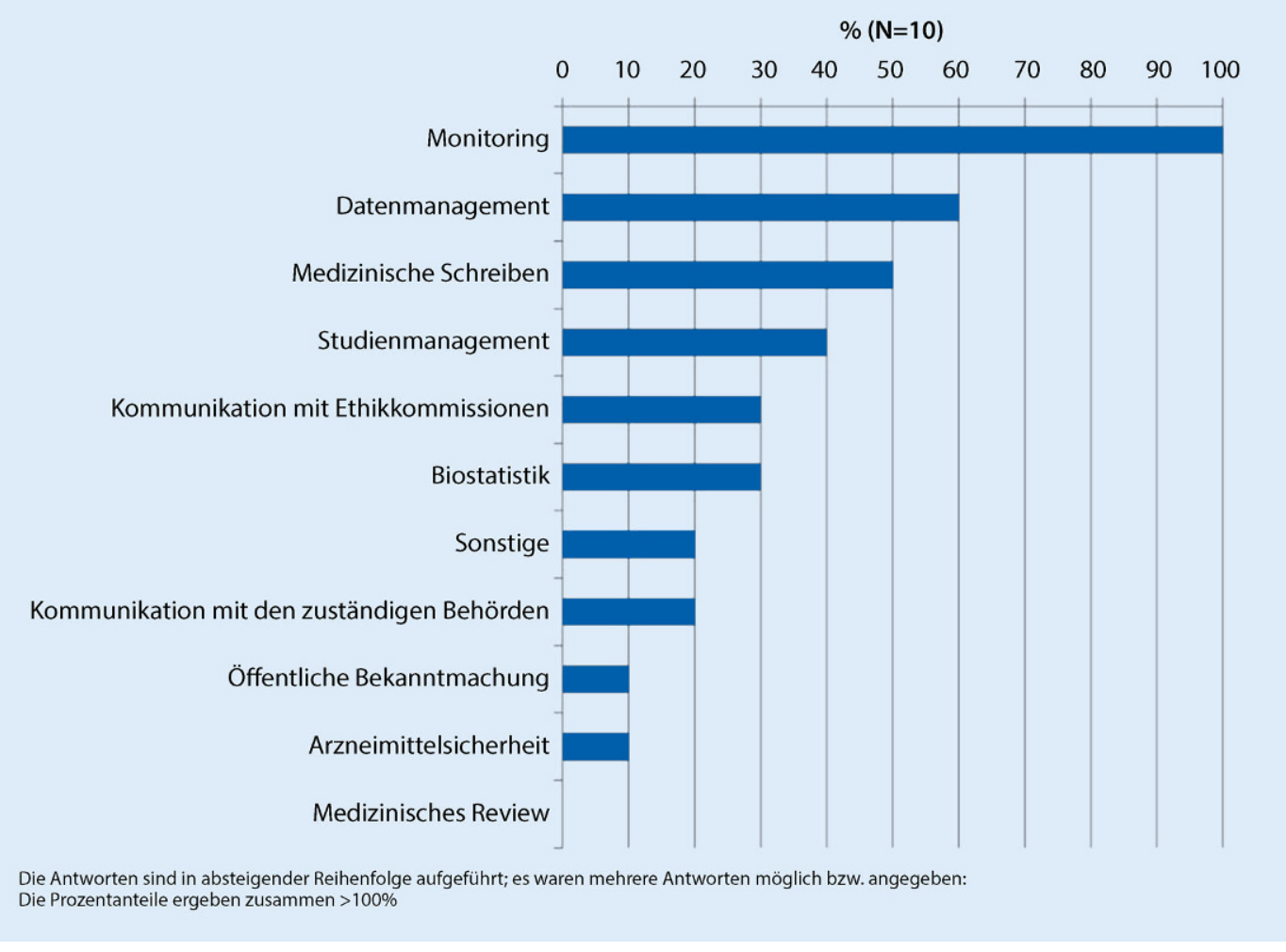

Abb. $1 \varangle$ Häufigkeiten des Outsourcing einzelner Studienleistungen an CROs. (৫ 2017 Hennig et al. [1])

schen Union (EU) hat: Damit eine klinische Prüfung mit Arzneimitteln in Deutschland durchgeführt werden kann, muss der Sponsor oder sein gesetzlicher Vertreter seinen Sitz in einem Mitgliedsstaat der Europäischen Union (EU) bzw. in einem anderen Vertragsstaat des Abkommens über den Europäischen Wirtschaftsraum (EWR) haben. Die Grundlage hierfür ist $\S 40$ Absatz 1 Satz 3 Nummer 1 Arzneimittelgesetz. Im Laufe der Zeit ist die Anzahl der medizinischen Auftragsinstitute stark gewachsen, um die zunehmende Nachfrage zu bedienen. Viele dieser CROs sind global organisiert und als große Unternehmen aufgestellt. Allein in Deutschland sind zurzeit 45 CROs Mitglieder des 1991 gegründeten Bundesverbands Medizinischer Auftragsinstitute (BVMA; www.bvma.de). Dieser 1991 gegründete Verband vertritt damit etwa 6000 Mitarbeitende bei CROs in Deutschland (Angaben des BVMA auf Anfrage vom April 2021). Im Jahr 2017 analysierte der Verband forschender Arzneimittelhersteller (vfa) mit einer Umfrage unter seinen deutschen Mitgliedern die aktuelle Praxis der Sponsoren, Tätigkeiten an CROs auszulagern. In der Umfrage sollten sowohl Art und Umfang der ausgelagerten Tätigkeiten erfasst werden als auch die Maßnahmen zur Überwachung der CROs [1]. Daraus wurde deutlich, dass das Monitoring die am häufigsten delegierte Aufgabe ist, die im Sinne eines umfassenden Outsourcings an bevorzugte Partner-CROs übertragen wird (s. - Abb. 1). Von den Aufsichtsbehörden European Medicines Agency (EMA) und Food and Drug Administration (FDA) wird ein umfassendes Aufsichtsprogramm von Sponsoren erwartet. Weiterführende Informationen zur Situation der Konstellation Industriesponsoren/CROs sowie ein jährliches Outsourcing Survey (Übersicht über ausgelagerte Tätigkeiten) finden sich unter https://www.pharmoutsourcing.com. Gleichzeitig gibt es erstaunlich wenig wissenschaftliche Untersuchungen darüber, welche Auswirkungen die Einbindung von CROs in klinische Studien hat, z. B. auf die Zeitrahmen des Vertragsabschlusses [2].

\section{Komplexität der Arbeitswelt in klinischen Studien}

In den letzten 2 Jahrzehnten ist der administrative Aufwand, den Studienzentren in Studien betreiben und mittragen müssen, erheblich gewachsen, und die Komplexität der Prüfpläne hat ebenfalls zugenommen. Prüfgruppen von mehr als 20 ärztlichen und nichtärztlichen Mitgliedern pro Studienzentrum sind in augenheilkundlichen Studien keine Seltenheit. Die Strukturen der Studienbegleitung und-überwachung passten sich an, und so haben die Studienteams nicht nur eine zusätzliche Schnittstelle in der Zusammenarbeit mit einer $\mathrm{CRO}$, sondern viele: unter anderem gehören dazu unmasked und masked Monitore (für verblindete und nicht verblindete Mitglieder des Studienteams) (s. - Abb. 2), On-site- (Besuche vor Ort) und Remote(die nur per Telefon/E-Mail mit dem Studienzentrum kommunizieren) Monitore; beauftragte Mitarbeiter der CRO für Vertragserstellung, Rechnungsvorbereitung, Rechnungserstattung. Daraus wird bereits ersichtlich, dass auch eine gute und vollständige Kommunikation innerhalb der CRO 
ein wesentlicher Punkt ist, welcher die Arbeit des Studienzentrums in der Studie erleichtern kann. Häufige Monitorwechsel im Verlauf einer Studie sind die Regel und bilden zusätzliche Quellen von Aufwand und Problemen für die Studienzentren, wenn Übergaben zwischen den Monitoren in kurzer Zeit oder unvollständig erfolgen oder wenn bereits überprüfte Originaldaten von einem nachfolgenden Monitor doppelt überprüft werden.

\section{Entwicklung eines Fragebogens der Arbeitsgemeinschaft (AG) Klinische Studienzentren der DOG}

Uns ist bislang keine Untersuchung bekannt, welche die Zusammenarbeit mit Auftragsinstituten in klinischen Studien aus der Perspektive der Studienzentren analysiert.

Die 2011 gegründete Arbeitsgemeinschaft Klinische Studienzentren der DOG [3] möchte sich den oben genannten Themen stellen und hat eine systematische Erfassung der Qualität in der Zusammenarbeit der Studienzentren mit CROs in multi- zentrischen, augenheilkundlichen Studien vorbereitet. Ein Fragebogen wurde entwickelt, der die Erfahrungen der einzelnen Zentren erfassen soll. Die Erfahrungen des Studienteams werden von einem vom Prüfarzt beauftragten Mitglied der Prüfgruppe (z. B. Studienkoordinator/in als Hauptkontaktperson an der Schnittstelle zur (RO) zusammengetragen. Es ist geplant, dass die Leiter der jeweiligen klinischen Studie die Ergebnisse sammeln und analysieren. Das Ergebnis wird an die jeweiligen Auftragsinstitute ebenso wie an die sie beauftragenden Sponsoren weitergegeben. Dadurch kann in der Zukunft zur Qualität der Unterstützung durch CROs und damit zur Studienqualität beigetragen werden. Unsere vorliegende, erste Arbeit zu diesem Projekt stellt vor, welche Bereiche der Zusammenarbeit im Fragebogen erfasst und analysiert werden.

\section{Fragebogen-Eckpunkte (s. auch} Zusatzmaterial online)

Der Fragebogen zur Erfassung von Erfahrungen in der Zusammenarbeit mit CROs umfasst folgende Bereiche:

- allgemeine Organisation/Verwaltung der CRO, aufgeteilt nach Projektleitung, Vertragsgestaltung, Rechnungsvorbereitung und Einhaltung von Zahlungszielen,

- Erreichbarkeit der Monitore,

- studienspezifische Kompetenz der Monitore,

- fachliche Kompetenz der Monitore,

- Rückmeldung der Monitore, zeitlich und inhaltlich,

- Absprachen zwischen „On-Site“- und "Remote"-Monitor,

- Zeitökonomie und Effizienz der Monitorkontakte,

- Monitorwechsel/-übergaben,

- Umsetzung von Anregungen/ Verbesserungsvorschläge.

Bei allen Fragen wird zusätzlich zwischen maskierten und unmaskierten, On-site-

Hier steht eine Anzeige. 


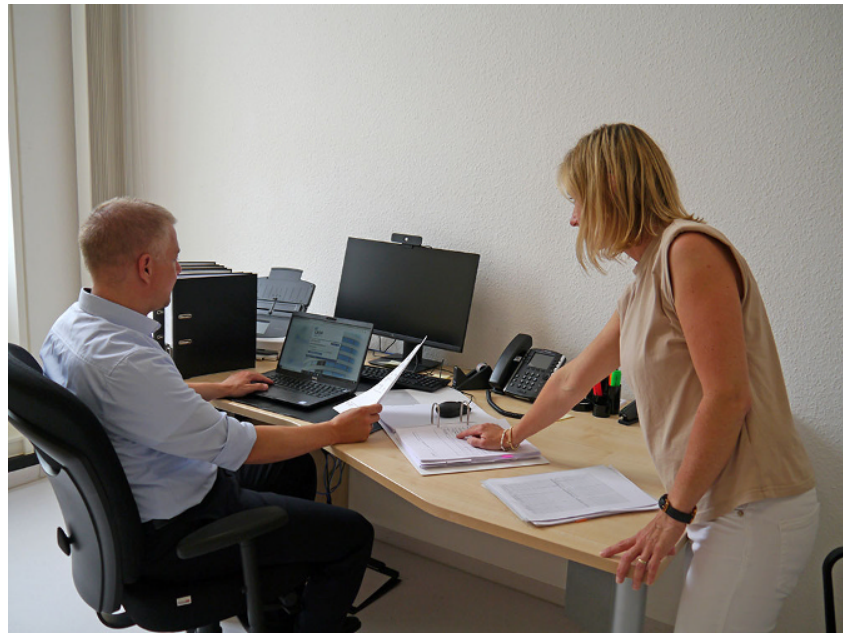

Abb. $2 \triangleleft$ Monitoring durch eine $C R O$ an einem klinischen Studienzentrum

und Remote-Monitoren differenziert. Der Fragebogen wird ab 2021 am Ende jeder multizentrischen Studie eingesetzt werden, um ein umfassendes Bild der Situation an deutschen Studienzentren zu erhalten. Nach Vorliegen der ersten Erfahrungen wird die AG DOG Klinische Studienzentren über die Ergebnisse berichten. Ziel des Fragebogens ist die langfristige Qualitätsverbesserung der Zusammenarbeit mit CROs und Sponsoren. Der Fragebogen soll hier die nötige Transparenz schaffen und bereits im Vorfeld kommuniziert werden.

\section{Fazit für die Praxis}

- Die Arbeitswelt in klinischen Studien hat sich für Studienzentralen an Augenkliniken, die sich an Studien beteiligen, in den letzten 20 Jahren massiv verändert.

- Zunehmend werden zahlreiche Aufgaben von Industriesponsoren an medizinische Auftragsinstitute (CROs) delegiert.

- Die Unterstützung durch die CRO hat groBe Bedeutung für den organisatorischen Aufwand, der an den Kliniken zur Durchführung einer Studie getrieben werden muss.

- Die AG DOG Klinische Studienzentren hat einen Fragebogen entwickelt, der in künftigen multizentrischen Studien eingesetzt wird.

- Dieser Fragebogen erfasst die Erfahrungen in der Zusammenarbeit mit der CRO, wird von den Leitern der klinischen Prüfung (LKPs) analysiert, und die Ergebnisse werden an CRO und Sponsor weitergegeben.

- Daraus werden sich Ansatzpunkte für weitere Verbesserungen der Zusammenarbeit zur Förderung der Studienqualität ergeben.

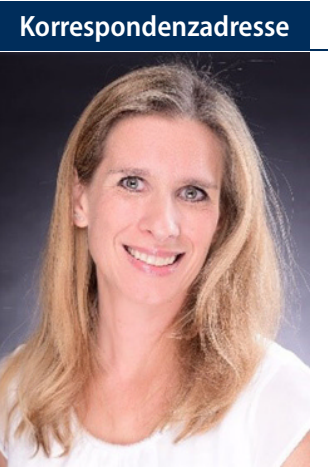

PD Dr.med. Katrin Lorenz, FEBO

Augenklinik und Poliklinik, Universitätsmedizin der Johannes Gutenberg-Universität Mainz Langenbeckstr. 1, 55131 Mainz, Deutschland katrin.lorenz@unimedizin-mainz.de

Mitglieder der Arbeitsgemeinschaft DOG Klinische Studienzentren. Tobias Peters (STZ eyetrial am Department für Augenheilkunde, ElfriedeAulhorn-Str. 7, 72076 Tübingen); Amelie Pielen (AuNeuberg-Str. 1, 30625 Hannover); Daniel Böhringer (Klinik für Augenheilkunde, Universitätsklinikum Freiburg und Medizinische Fakultät der Albert-LudwigsUniversität Freiburg, Killianstr. 5, 79106 Freiburg); Dorina Seger (Klinik für Augenheilkunde, Universitätsklinikum Freiburg und Medizinische Fakultät der Albert-Ludwigs-Universität Freiburg, Killianstr. 5, 79106 Freiburg); Fabian Wozar (Department für Augenheilkunde, Elfriede-Aulhorn-Str. 7, 72076 Tübingen); Stefanie Otto (Klinik für Augenheilkunde, Baldingerstraße, 35043 Marburg); Kerstin Petermann (Klinik für Augenheilkunde, Studienzentrale, Theodor-Stern-Kai 7, Haus 7A, 2. OG, 60590 Frankfurt am Main); Fanni Molnar (Klinik für Augenheilkunde, Universitätsklinikum Freiburg und Medizinische Fakultät der Albert-Ludwigs-Universität Freiburg, Killianstr. 5, 79106 Freiburg); Frank Holz (UniversitätsAugenklinik Bonn, Ernst-Abbe-Str. 2, 53127 Bonn); Frank Schüttauf (Univ.-Augenklinik, Martinistr. 52, 20246 Hamburg); leva Laure (Augenklinik und Poliklinik der Universitätsmedizin Mainz, Johannes genklinik Medizinische Hochschule Hannover, Carl-
Gutenberg-Universität, Langenbeckstr. 1, 55131 Mainz); Anna Beck (Augenklinik und Poliklinik der Universitätsmedizin Mainz, Johannes GutenbergUniversität, Langenbeckstr. 1, 55131 Mainz); Luise Fornoff (Augenklinik Ludwigshafen, Bremserstr. 79, 67063 Ludwigshafen); Frau Dona (Klinik für Augenheilkunde Studienzentrale, Theodor-Stern-Kai 7, Haus 7A, 2. OG, 60590 Frankfurt am Main); Lisa Hinzelmann (Klinik für Augenheilkunde, Studienzentrale, Theodor-Stern-Kai 7, Haus 7A, 2. OG, 60590 Frankfurt am Main); Raffael Liegl (Universitäts-Augenklinik Bonn, Ernst-Abbe-Str. 2, 53127 Bonn); Simon Dulz (Univ.-Augenklinik, Martinistr. 52, 20246 Hamburg); Carsten Grohmann (Univ.-Augenklinik, Martinistr. 52, 20246 Hamburg); Stephanie Winterhalder (Klinik für Augenheilkunde, Universitätsklinikum Freiburg und Medizinische Fakultät der Albert-Ludwigs-Universität Freiburg, Killianstr. 5, 79106 Freiburg); Katharina Allgeier (Klinik für Augenheilkunde, Universitätsklinikum Freiburg und Medizinische Fakultät der AlbertLudwigs-Universität Freiburg, Killianstr. 5, 79106 Freiburg); Dimitrij Subottin (Augenklinik und Poliklinik der Universitätsmedizin Mainz, Johannes GutenbergUniversität, Langenbeckstr. 1, 55131 Mainz); Anna Mangold (Universitätsmedizin Göttingen, Klinik für Augenheilkunde, Robert-Koch-Str. 40, 37075 Göttingen); Lars Choritz (Otto-von-Guericke-Universität, Universitätsklinikum Magdeburg A. ö. R., Leipziger Str. 44, 39120 Magdeburg); Stefan Kreis (Klinik für Augenheilkunde, Universitätsklinikum Essen, Hufelandstr. 55, 45147 Essen); Nicole Eter (Universitätsklinikum Münster [UKM], Klinik für Augenheilkunde, Albert-Schweitzer-Campus 1, Gebäude D15, 48149 Münster); Ulrich Kellner (AugenZentrum Siegburg, MVZ ADTC GmbH Siegburg, Europaplatz 3, 53721 Siegburg); Monica Lang (Augenklinik, Heidelberger Landstr. 379, 64297 Darmstadt); Andreea Gamulescu (Univ.-Augenklinik Regensburg, Franz-JosefStrauß-Allee 11, 93053 Regensburg); Svenja Tamm (Univ.-Augenklinik Regensburg, Franz-Josef-Strauß Allee 11, 93053 Regensburg); Focke Ziemssen (Department für Augenheilkunde, Elfriede-AulhornStr. 7, 72076 Tübingen); Katarzyna Kallus (Universitätsmedizin Göttingen, Klinik für Augenheilkunde, Robert-Koch-Str. 40, 37075 Göttingen); Sabine Aisenbrey (Klinik für Augenheilkunde im Vivantes Klinikum Neukölln, Rudower Str. 48, 12351 Berlin Neukölln); Immanuel Seitz (Department für Augenheilkunde, Elfriede-Aulhorn-Str. 7, 72076 Tübingen); Tanja Lengnink (STZ eyetrial am Department für Augenheilkunde, Elfriede-Aulhorn-Str. 7, 72076 Tübingen); Nadine Kahle (Department für Augenheilkunde, ElfriedeAulhorn-Str. 7, 72076 Tübingen); Anna Bajor (Augenklinik Medizinische Hochschule Hannover, Carl-Neuberg-Str. 1, 30625 Hannover); Bettina von Livonius (Univ.-Augenklinik, Mathildenstr. 8, D-80336 München); Matus Rehak (Univ.-Augenklinik, Liebigstr. 12, Haus 1, 04103 Leipzig); Rainer Guthoff (Augenklinik Universitätsklinikum Düsseldorf, Moorenstr. 5, 40225 Düsseldorf); Martina Hofmann (UniversitätsAugenklinik Bonn, Ernst-Abbe-Str. 2, 53127 Bonn); Christoph Ehlken (Klinik für Ophthalmologie des UKSH, Campus Kiel, Arnold-Heller-Str. 3, 24105 Kiel); Andrea Koschinski (Augenzentrum am St. FranziskusHospital, Hohenzollernring 74, 48145 Münster); Georg Spital (Augenzentrum am St. Franziskus-Hospital, Hohenzollernring 74, 48145 Münster); Marc Schargus (Asklepios Augenklinik Nord-Heidberg, Hamburg, Deutschland; Klinik für Augenheilkunde, HeinrichHeine-Universität, Düsseldorf); Ramin Khoramnia (Universitätsklinikum Heidelberg, Augenklinik, Im Neuenheimer Feld 400, 69120 Heidelberg); Philip Enders (Univ.-Augenklinik Köln, Kerpener Str. 62, 50937 Köln); Lebriz Altay (Univ.-Augenklinik Köln, 
Kerpener Str. 62, 50937 Köln); Beate Prinz (Univ.Augenklinik Köln, Kerpener Str. 62, 50937 Köln)

Funding. Open Access funding enabled and organized by Projekt DEAL.

\section{Einhaltung ethischer Richtlinien}

Interessenkonflikt. A. Adorf, K. Lorenz, N. Feltgen und B. Wilhelm geben an, dass kein Interessenkonflikt besteht.

Für diesen Beitrag wurden von den Autoren keine Studien an Menschen oder Tieren durchgeführt. Für die aufgeführten Studien gelten die jeweils dort angegebenen ethischen Richtlinien.

Open Access. Dieser Artikel wird unter der Creative Commons Namensnennung 4.0 International Lizenz veröffentlicht, welche die Nutzung, Vervielfältigung, Bearbeitung, Verbreitung und Wiedergabe in jeglichem Medium und Format erlaubt, sofern Sie den/die ursprünglichen Autor(en) und die Quelle ordnungsgemäß nennen, einen Link zur Creative Commons Lizenz beifügen und angeben, ob Änderungen vorgenommen wurden.

Die in diesem Artikel enthaltenen Bilder und sonstiges Drittmaterial unterliegen ebenfalls der genannten Creative Commons Lizenz, sofern sich aus der Abbildungslegende nichts anderes ergibt. Sofern das betreffende Material nicht unter der genannten Creative Commons Lizenz steht und die betreffende Handlung nicht nach gesetzlichen Vorschriften erlaubt ist, ist für die oben aufgeführten Weiterverwendungen des Materials die Einwilligung des jeweiligen Rechteinhabers einzuholen.

Weitere Details zur Lizenz entnehmen Sie bitte der Lizenzinformation auf http://creativecommons.org/ licenses/by/4.0/deed.de.

\section{Literatur}

1. Hennig M, Hundt F, Busta S et al (2017) Current practice and perspectives in CRO oversight based on a survey performed among members of the German association of research-based pharmaceutical companies (vfa). Ger Med Sci 26:15

2. Gobbini E, Pilotto S, Pasello G et al (2018) Effect of contract research organization bureaucracy in clinical trial management: a model from lung cancer. Clin Lung Cancer 19(2):191-198

3. Deutsche Ophthalmologische Gesellschaft (DOG) (2019) Recommendations by the DOG for the planning of clinical trials on behalf of third parties. Ophthalmologe 116:21-24

\section{Collaboration with clinical research organizations. Introduction of a questionnaire for clinical study centers}

Clinical trials with pharmaceuticals or medical devices make complex demands on sponsors and participating centers. During the past two decades, sponsors have increasingly delegated regulatory and organizational tasks to clinical research organizations (CRO). As a rule, these companies are the main interface for the collaboration with the participating study centers. The main purpose of the participation is the support of the study centers for achieving an optimal study quality. The study centers involved in the DOG working group on clinical study centers perceived varying experiences in the collaboration with CROs. In the future these experiences will be systematically assessed at the participating study centers and analyzed by the coordinating investigator. Reflecting these experiences to the respective CROs and the delegating sponsors will contribute to the quality of support by CROs and herewith to the quality of clinical trials. This paper presents which areas of collaboration will be assessed and analyzed.

\section{Keywords}

Clinical trials $\cdot$ CRO $\cdot$ DOG $\cdot$ Study quality $\cdot$ Questionnaire 